\title{
Determinants of Overall Knowledge and Health Behaviors Towards Hepatitis B and C Among Ever- Married Women in Pakistan: Evidence Based on Demographic and Health Survey 2017-18
}

\section{Sidra Maqsood}

Government College University Lahore

\section{Sarosh lqbal}

University of the Punjab

Rubeena Zakar

University of the Punjab

Muhammad Zakria Zakar

University of Okara

Florian Fischer ( $\square$ florian.fischer1@charite.de)

Charite Universitatsmedizin Berlin https://orcid.org/0000-0002-4388-1245

\section{Research article}

Keywords: HBV, HCV, Knowledge, Attitude, Practice, Social determinants

Posted Date: November 17th, 2020

DOI: https://doi.org/10.21203/rs.3.rs-105800/v1

License: (c) (1) This work is licensed under a Creative Commons Attribution 4.0 International License.

Read Full License 


\section{Abstract}

Background: In 2019, around 5 million and 10 million people were affected by hepatitis B virus (HBV) and hepatitis C virus (HCV) respectively in Pakistan. On World Hepatitis Day 2019, Pakistan's Government announced the Prime Minister's Plan to eliminate HBV and HCV from the country by 2030. In order to achieve this goal, adequate knowledge about HBV and HCV regarding mode of transmission, symptoms of the disease, and awareness about available treatments and vaccines is imperative. The present study aims to investigate the determinants related to overall knowledge about and behaviour in relation to HBV and HCV amongst married women in Pakistan.

Methods: Secondary data analysis was carried out using the Pakistan Demographic and Health Survey (PDHS) 2017-18. A series of questions regarding women's knowledge about how to avoid HBV and HVC and their health behaviour in relation to HBV and HCV were posed to 12,364 ever-married women of reproductive age (15-49 years). Bivariate and multivariable logistic regression was applied to examine the effects of socio-demographic characteristics and covariates on women's overall knowledge and health behaviour regarding HBV and HCV.

Results: The findings highlight that the majority of women (88.3\%) have heard of HBV and HCV. Nonetheless, only $34.8 \%$ had comprehensive knowledge about how to avoid HBV and HCV. Few women (11.3\%) had been tested for HBV or HCV during the year preceding the survey. Furthermore, the results indicated that women from Sindh, living in urban areas, aged 35 years and over, having more than 10 years of schooling, belonging to the richest wealth quintile and working as professionals or in clerical/sales \& service jobs reported better knowledge and health behaviours regarding HBV and HCV.

Conclusion: This study provides evidence that women's socio-demographic characteristics create differences in their overall knowledge about and attitudes towards HBV and HCV. This research emphasized that there is a need to create awareness about the causes and prevention of HBV and HCV in order to achieve the goal of eliminating these diseases in Pakistan by 2030.

\section{Background}

Hepatitis $B$ and $C$ infections cause a range of ailments, ranging from asymptomatic to life-threatening disease [1]. According to estimates by the World Health Organization (WHO), globally 325 million people are affected by hepatitis $B$ virus (HBV) and hepatitis $C$ virus $(\mathrm{HCV})$, resulting in 1.4 million deaths across the world per year. The WHO's data shows that around 5 million and 10 million people were affected by HBV and HCV respectively in Pakistan during 2019 [2]. A lack of preventive measures, testing services, or treatment resources, the use of unsterilized invasive medical instruments and transfusions of infected blood are the leading causes of high HBV and HCV rates [2,3]. Furthermore, the data suggests that the primary cause of hepatocellular carcinoma is either hepatitis C or hepatitis B in Pakistan [4]. In October 2017, following the WHO's Global Health Sector Strategy (GHSS), Pakistan's Government launched the first National Hepatitis Strategic Framework (2017-2021), aiming to reduce the incidence and chronic 
cases of hepatitis B and C by $10 \%$ and $30 \%$, respectively $[2,5]$. In addition to this, on World Hepatitis Day 2019, Pakistan's Government also announced the Prime Minister's Plan to eliminate HBV and HCV from the country by 2030 . Through this plan, the federal government aims to facilitate provincial governments in providing leadership and coordination to scale up preventive, testing and treatment services for hepatitis [2].

Despite the governmental efforts and commitment, HBV and HCV are still serious public health challenges in Pakistan. Adequate knowledge about HBV and HCV, regarding mode of transmission, symptoms of disease and awareness about available treatments and vaccines is very important for encouraging a favourable attitude towards seeking treatment and avoiding the spread of infections [6, 7]. A previous study conducted in Pakistan indicated that married women had poor and incorrect knowledge about the causes, prevention and treatment of HBV [8]. It is evident through research findings that pregnant women are more at risk, if infected, of transmitting viral hepatitis $B$ and $C$ via sexual, vertical (mother to child during childbirth) and horizontal routes (e.g., blood transfusions and contaminated injections). Therefore, married women's knowledge about HBV and HCV is crucial for controlling the disease $[9,10]$.

In light of the above context, there is a dire need for research to understand the determinants of overall knowledge about HBV and HCV among married women. Women who have adequate knowledge about the causes, prevention and treatment of HBV and HCV can be expected to take precautionary measures to avoid the spread of disease and to access treatment services. Previous studies suggest that, in Pakistan, hepatitis B patients lack information about attitudes and practices regarding the prevention of disease [6], and that women have less knowledge about HBV and HCV than men [11]. However, the findings of a study conducted among medical students in Pakistan revealed that female students have more diagnostic knowledge about HBV and HCV than male students [12]. The findings of another study with male respondents revealed that respondents have low levels of knowledge about HBV and HCV, which leads to unfavourable attitudes towards the practising of preventive measures [13].

The studies referred to above with reference to Pakistan were carried out with small sample sizes and lacked evidence of married women's knowledge and behaviour regarding HBV and HCV. Hence, the present study aims to bridge this research gap, based on a larger sample that is representative of the population of Pakistan. Its prime objective is to investigate various determinants affecting overall knowledge and behaviour relating to HBV and HCV amongst married women in Pakistan.

\section{Theoretical framework}

The theoretical foundation of this research draws upon knowledge, attitude, practice (KAP) theory, which combines aspects of knowledge, attitudes and practice [14], and the WHO's framework of social determinants of health [15].

The assumptions of KAP theory indicate that modifications occur in the health behaviour of individuals after seeking health education and knowledge. It provides a rational model linking health education and 
behavioural changes [16]. The core postulate of KAP theory emphasizes three successive stages through which an individual's behaviour passes in order to adapt to changes. These stages are: acquisition of related knowledge, attitude modification and formation of behaviour. Furthermore, KAP theory postulates that acquiring sufficient knowledge regarding symptoms, causes and ways to prevent disease inculcates positive attitudes among individuals about following preventive methods and seeking medical care. Several previous studies have reported that individuals' KAP level was significantly linked to addressing false perceptions, management of illness, seeking healthcare services and embracing disease-preventive behaviour [17-21].

Furthermore, the social determinants of the health framework emphasize the role of individuals' sociodemographic factors in determining their health. It indicates that individuals' health status may vary depending upon their sociodemographic characteristics [22]. The findings of past studies indicate that low socioeconomic strata are linked with low levels of treatment compliance, the inaccessibility of healthcare facilities and insufficient ability to afford medical treatment [23-26]. The literature also reveals that gender, age, level of education and income affect an individual's knowledge and behaviour in terms of seeking good health $[27,28]$.

Based on the postulates of KAP theory and the social determinants of health framework, this research explored the social factors linked to the knowledge and behaviour of ever-married women in Pakistan regarding $\mathrm{HBV}$ and $\mathrm{HCV}$.

\section{Methods}

\section{Study design}

This research conducted secondary data analysis, using the fourth and latest Pakistan Demographic and Health Survey (PDHS) 2017-18. Currently, PDHS 2017-18 provides the largest national representative estimates of demographic and health indicators in Pakistan. Furthermore, it provides the largest dataset of variables related to HBV and HCV knowledge among the general population. PDHS 2017-18 is a cross-sectional survey, adopting a stratified two-stage sample design. The stratification was based on urban and rural areas, and a total of 16 strata were obtained, consisting of eight strata each for urban and rural areas. During the first stage, 580 clusters consisting of enumeration blocks were selected, while in the second stage, 16,240 households ( 28 households per cluster) were selected, using a probability systematic selection process.

PDHS 2017-18 collected data on different types of questionnaires. In the present study, data obtained through the women's questionnaire was used. The women's questionnaire was administered to 12,364 ever-married women of reproductive age (15-49 years). This women's questionnaire included questions regarding women's knowledge and behaviour regarding hepatitis $\mathrm{B}$ and $\mathrm{C}$.

\section{Outcome variables}


In this study, women's awareness, knowledge/health beliefs about HBV or HCV, and health behaviour are taken as outcome variables. Initially, women' awareness was explored, if they had ever heard of illnesses called HBV or HCV ("Yes"/"No"). Women who replied "yes" were asked further questions to inquire about their knowledge and health behaviour regarding HBV or HCV.

Women's overall knowledge or health beliefs about HBV or HCV was inferred from the following question: "Is there anything a person can do to avoid getting HBV or HCV?" ("Yes"/"No"/“Don't know"). Women who replied "yes" were further asked: "What can a person do to avoid getting HBV or HCV?" Possible responses to this question included six options: "practice safe sex", "safe blood transfer", "use disposable syringe", "avoid contaminated food/water", "avoid contact with infected person", and "ensure dentists' instruments properly sterilized". Respondents who replied correctly were coded as 1, while those who replied incorrectly or "don't know" were coded as 0 . Hence, the score for women's knowledge about HBV or $\mathrm{HCV}$ ranged from 0 to 6 . This score was dichotomized into no knowledge (score 0 ) vs. some knowledge (score 1-6).

Health behaviour was determined through the following question: 1) "Have you ever been tested for HBV or HCV?" ("Yes"/“No"). 2) Respondents who reported "yes" were asked about their recent test: "How many months ago was your most recent test for HBV or HCV?" ("Within the last year"/"More than one years ago").

\section{Independent variables}

In this study, women's healthcare decision-making autonomy, exposure to mass media to access information, health insurance coverage and the accessibility of distant healthcare facilities were taken as independent variables, which may influence women's knowledge and behaviour about HBV or HCV.

Women's healthcare autonomy measures their overall contribution towards decisions about healthcare ("Who usually decides about your healthcare?"). Possible responses were: "respondent alone", "husband/partner alone", "respondent and husband/partner jointly", "respondent and other person", and "someone else or others". For this study, responses to the above question were dichotomized into one of two categories: whether the woman has "a say at all" (either alone or jointly with her husband/partner or jointly with another person) or whether she has "no say at all" (in cases where her husband/partner alone, someone else or others make the decisions). Exposure to mass media to access information measures the women's frequency of reading a newspaper, watching TV or listening to the radio, and was grouped into two categories ("Yes"/“No"). Furthermore, women were asked about health insurance coverage ("Yes"/“No") and the accessibility of distant healthcare facilities for seeking medical services ("big problem"/“not a big problem").

A set of sociodemographic variables was included: geographical classification ("urban"/"rural"), region/province (Punjab; Sindh; Khyber Pakhtunkhwa; Baluchistan; Gilgit Baltistan; Islamabad), age of respondents (in years), educational status of respondents and husbands, employment/occupation of respondents and husbands, household wealth quintile. Women's age was grouped into three categories 
("15-24 years"/“25-34 years"/“35 years and above”). The educational status of women and their spouses/husbands was grouped into four categories ("uneducated/no formal schooling"/"up to 5 years of schooling"/"6-10 years of schooling"/"more than 10 years of schooling"). The employment/occupation of respondents and their spouses/husbands was grouped into four categories ("not working or unemployed"/"professional, clerical, sales \& services"/ "agriculture"/"manual or household worker").

\section{Statistical analysis}

SPSS version 21 was used for the data analysis. We applied sampling weights for all analyses. Descriptive statistics were employed for sociodemographic characteristics. Measures of women's awareness, overall knowledge and health behaviour regarding hepatitis $\mathrm{B}$ or $\mathrm{C}$, women's healthcare decision-making autonomy, exposure to mass media and health insurance coverage were compiled in the form of frequencies and percentages. Multicollinearity between variables was examined using a variance inflation factor (VIF). However, no multicollinearity (VIF $\geq 10$ ) was observed.

Bivariate and multivariable logistic regression models were applied to investigate the effect of sociodemographic characteristics and further independent variables on women's overall knowledge related to HBV and HCV. The results are presented as odds ratios (OR) and adjusted odds ratios (AOR) with $95 \%$ confidence intervals $(\mathrm{Cl})$.

\section{Results}

\section{Sociodemographic characteristics and covariates}

Table 1 describes the sociodemographic characteristics and covariates of the sample of 12,364 evermarried women. The majority resided in rural areas (63.2\%) and belonged to the age group 25-34 years $(40.1 \%)$. With reference to educational status, the majority of respondents $(49.2 \%)$ had no formal schooling, while $21.2 \%$ had completed $6-10$ years of schooling, and only $13.1 \%$ had attained more than 10 years of schooling. The majority of respondents (80.1\%) were unemployed. Most of the respondents (66\%) had access to mass media and half (50.5\%) reported that they had autonomy in making healthcare decisions. Furthermore, a significant percentage $(42.0 \%)$ of respondents reported that the accessibility of distant healthcare facilities for seeking medical services was a problem, and almost all respondents (98.6\%) had no health insurance coverage. 
Table 1

Sample description $(n=12,364)$

\begin{tabular}{|c|c|c|}
\hline Characteristics & $\mathbf{n}$ & $\%$ \\
\hline \multicolumn{3}{|l|}{ Sociodemographic characteristics } \\
\hline \multicolumn{3}{|l|}{ Regions/Provinces } \\
\hline Punjab & 6,630 & 53.6 \\
\hline Sindh & 2,850 & 23.1 \\
\hline Baluchistan & 642 & 5.2 \\
\hline Khyber Pakhtunkhwa & 1,901 & 15.4 \\
\hline Islamabad Capital Territory & 107 & 0.9 \\
\hline FATA & 234 & 1.9 \\
\hline \multicolumn{3}{|l|}{ Geographical classification } \\
\hline Urban & 4,550 & 36.8 \\
\hline Rural & 7,814 & 63.2 \\
\hline \multicolumn{3}{|l|}{ Respondent's age } \\
\hline $15-24$ years & 2,489 & 20.1 \\
\hline $25-34$ years & 4,961 & 40.1 \\
\hline 35 years and above & 4,915 & 39.8 \\
\hline \multicolumn{3}{|l|}{ Respondent's educational status } \\
\hline No formal schooling & 6,080 & 49.2 \\
\hline Up to 5 years of schooling & 2,097 & 16.5 \\
\hline $6-10$ years of schooling & 2,623 & 21.2 \\
\hline More than 10 years of schooling & 1,624 & 13.1 \\
\hline \multicolumn{3}{|l|}{ Respondent's Occupation } \\
\hline Not working / unemployed & 9,894 & 80.1 \\
\hline Professional, clerical, sales \& services & 648 & 5.2 \\
\hline Agriculture & 778 & 6.3 \\
\hline Manual or Household worker & 1,034 & 8.4 \\
\hline \multicolumn{3}{|l|}{ Husband's educational status } \\
\hline No formal schooling & 3,480 & 29.5 \\
\hline
\end{tabular}




\begin{tabular}{|c|c|c|}
\hline Characteristics & $\mathbf{n}$ & $\%$ \\
\hline Up to 5 years of schooling & 1,840 & 15.6 \\
\hline $6-10$ years of schooling & 4,165 & 35.3 \\
\hline More than 10 years of schooling & 2,310 & 19.6 \\
\hline \multicolumn{3}{|l|}{ Husband's occupation } \\
\hline Not working / unemployed & 489 & 4.1 \\
\hline Professional, clerical, sales \& services & 3,980 & 33.7 \\
\hline Agriculture & 2,013 & 17.1 \\
\hline Manual or household worker & 5,324 & 45.1 \\
\hline \multicolumn{3}{|l|}{ Wealth quintile } \\
\hline Richest & 2,579 & 20.9 \\
\hline Richer & 2,594 & 21 \\
\hline Middle & 2,504 & 20.3 \\
\hline Poorer & 2,430 & 19.7 \\
\hline Poorest & 2,258 & 18.3 \\
\hline \multicolumn{3}{|l|}{ Covariates } \\
\hline \multicolumn{3}{|c|}{ Exposure to mass media / Access to information } \\
\hline Yes & 8,153 & 66.0 \\
\hline No & 4,200 & 34.0 \\
\hline \multicolumn{3}{|l|}{ Healthcare decision-making / Autonomy } \\
\hline Yes & 5,979 & 50.5 \\
\hline No & 5,850 & 49.5 \\
\hline \multicolumn{3}{|c|}{ Accessibility to distant health facility for seeking medical services } \\
\hline Big problem & 5,191 & 42.0 \\
\hline Not a big problem & 7,164 & 58.0 \\
\hline \multicolumn{3}{|l|}{ Health insurance coverage } \\
\hline Yes & 177 & 1.4 \\
\hline No & 12,187 & 98.6 \\
\hline
\end{tabular}


The majority of respondents (88.3\%) were aware of HBV and HCV. With reference to women's knowledge of $\mathrm{HBV}$ and HCV, it was found that more than half of respondents $(57.4 \%)$ were aware of various ways to avoid HBV and HCV. However, only some of the respondents (34.8\%) were found to have comprehensive knowledge about how to avoid HBV and HCV (Table 2). Upon probing, the results showed that less than one-fifth of women reported contaminated water and food (17.9\%) or use of an infected syringe (12.8\%) as the main risk factors for HBV and HCV. Furthermore, some of the women were also found to be aware of safe sex practices (6.8\%), safe blood transfusion (9.4\%), and refraining from contact with an infected person (7.9\%) to avoid HBV and HCV (Fig. 1).

Table 2

Awareness, knowledge and health behavior regarding hepatitis $\mathrm{B}$ or $\mathrm{C}(\mathrm{n}=$ 12,364)

\begin{tabular}{|c|c|c|}
\hline Characteristics & $\mathbf{n}$ & $\%$ \\
\hline \multicolumn{3}{|c|}{ Women has ever heard of hepatitis B or C } \\
\hline Yes & 10,919 & 88.3 \\
\hline No & 1,445 & 11.7 \\
\hline \multicolumn{3}{|c|}{ Women's awareness about various ways to avoid hepatitis B or C } \\
\hline Yes & 4,654 & 57.4 \\
\hline No & 6,264 & 42.6 \\
\hline \multicolumn{3}{|c|}{ Women's comprehensive knowledge to avoid hepatitis B or C } \\
\hline Yes & 3,804 & 34.8 \\
\hline No & 7,114 & 65.2 \\
\hline \multicolumn{3}{|c|}{ Ever been tested for hepatitis B or C } \\
\hline Yes & 3,174 & 25.7 \\
\hline No & 7,745 & 62.6 \\
\hline \multicolumn{3}{|c|}{ Months ago tested for hepatitis B or C } \\
\hline Within last year & 1,437 & 45.3 \\
\hline More than one year ago & 1,735 & 54.7 \\
\hline
\end{tabular}


Table 3

Association of women's knowledge and health behavior on hepatitis B or C with sociodemographic characteristics and covariates $(n=12,364)$

\begin{tabular}{|c|c|c|c|c|c|c|}
\hline \multirow[t]{2}{*}{ Characteristics } & \multicolumn{3}{|c|}{$\begin{array}{l}\text { Knowledge about hepatitis B } \\
\text { or C }\end{array}$} & \multicolumn{3}{|c|}{$\begin{array}{l}\text { Health behavior about hepatitis } \\
\text { B or C }\end{array}$} \\
\hline & Yes & No & p-value* & Yes & No & p-value* \\
\hline \multicolumn{7}{|l|}{ Regions/provinces } \\
\hline Punjab & 34.2 & 65.8 & \multirow[t]{6}{*}{$<0.01$} & 29.8 & 70.2 & \multirow[t]{6}{*}{$<0.01$} \\
\hline Sindh & 41 & 59 & & 34.8 & 65.2 & \\
\hline Baluchistan & 28.3 & 71.7 & & 18.2 & 81.8 & \\
\hline Khyber Pakhtunkhwa & 31 & 69 & & 21.9 & 78.1 & \\
\hline Islamabad Capital Territory & 56.1 & 43.9 & & 38.8 & 61.2 & \\
\hline FATA & 17.6 & 82.4 & & 19 & 81 & \\
\hline \multicolumn{7}{|l|}{ Geographical classification } \\
\hline Urban & 46 & 54 & \multirow[t]{2}{*}{$<0.01$} & 38.1 & 61.9 & \multirow[t]{2}{*}{$<0.01$} \\
\hline Rural & 28 & 72 & & 23.5 & 76.5 & \\
\hline \multicolumn{7}{|l|}{ Respondent's age } \\
\hline $15-24$ years & 25.2 & 74.8 & \multirow[t]{3}{*}{$<0.01$} & 18.2 & 81.8 & \multirow[t]{3}{*}{$<0.01$} \\
\hline $25-34$ years & 35.8 & 64.2 & & 31.6 & 68.4 & \\
\hline 35 years and above & 38.4 & 61.6 & & 31.6 & 68.4 & \\
\hline \multicolumn{7}{|l|}{ Respondent's educational status } \\
\hline No formal schooling & 23.9 & 76.1 & & 22 & 78 & \\
\hline Up to 5 years of schooling & 33.4 & 66.6 & & 26.2 & 73.8 & \\
\hline $6-10$ years of schooling & 40.9 & 59.1 & & 33.1 & 66.9 & \\
\hline More than 10 years of schooling & 62.5 & 37.5 & & 48.8 & 51.2 & \\
\hline \multicolumn{7}{|l|}{ Respondent's occupation } \\
\hline Not working / unemployed & 35.1 & 64.9 & \multirow[t]{3}{*}{$<0.01$} & 29.1 & 70.9 & \multirow[t]{3}{*}{$<0.01$} \\
\hline $\begin{array}{l}\text { Professional, clerical, sales \& } \\
\text { services }\end{array}$ & 52.2 & 47.8 & & 41.8 & 58.2 & \\
\hline Agriculture & 16.9 & 83.1 & & 17.8 & 82.2 & \\
\hline
\end{tabular}




\begin{tabular}{|c|c|c|c|c|c|c|}
\hline \multirow[t]{2}{*}{ Characteristics } & \multicolumn{3}{|c|}{$\begin{array}{l}\text { Knowledge about hepatitis B } \\
\text { or C }\end{array}$} & \multicolumn{3}{|c|}{$\begin{array}{l}\text { Health behavior about hepatitis } \\
\text { B or C }\end{array}$} \\
\hline & Yes & No & p-value* & Yes & No & p-value* \\
\hline Manual or household worker & 33.6 & 66.4 & & 28.7 & 71.3 & \\
\hline \multicolumn{7}{|l|}{ Husband's educational status } \\
\hline No formal schooling & 23.8 & 76.2 & \multirow[t]{4}{*}{$<0.01$} & 19.9 & 80.1 & \multirow[t]{4}{*}{$<0.01$} \\
\hline Up to 5 years of schooling & 30.2 & 69.8 & & 27.6 & 72.4 & \\
\hline $6-10$ years of schooling & 35.3 & 64.7 & & 29.3 & 70.7 & \\
\hline More than 10 years of schooling & 53 & 47 & & 42.2 & 57.8 & \\
\hline \multicolumn{7}{|l|}{ Husband's occupation } \\
\hline Not working / unemployed & 29.4 & 70.6 & \multirow[t]{4}{*}{$<0.01$} & 23.8 & 76.2 & \multirow[t]{4}{*}{$<0.01$} \\
\hline $\begin{array}{l}\text { Professional, clerical, sales \& } \\
\text { services }\end{array}$ & 44.3 & 55.7 & & 37.3 & 62.7 & \\
\hline Agriculture & 24.2 & 75.8 & & 23 & 77 & \\
\hline Manual or household worker & 32.1 & 67.9 & & 25.6 & 74.4 & \\
\hline \multicolumn{7}{|l|}{ Wealth quintile } \\
\hline Richest & 55.3 & 44.7 & \multirow[t]{5}{*}{$<0.01$} & 43.9 & 56.1 & \multirow[t]{5}{*}{$<0.01$} \\
\hline Richer & 38.1 & 61.9 & & 33.6 & 66.4 & \\
\hline Middle & 32.2 & 67.8 & & 26.1 & 73.9 & \\
\hline Poorer & 23 & 77 & & 18.9 & 81.1 & \\
\hline Poorest & 19.9 & 80.1 & & 18.6 & 81.4 & \\
\hline \multicolumn{7}{|c|}{ Exposure to mass media / Access to information } \\
\hline Yes & 39.9 & 60.1 & \multirow[t]{2}{*}{$<0.01$} & 32.9 & 67.1 & \multirow[t]{2}{*}{$<0.01$} \\
\hline No & 24.1 & 75.9 & & 21.0 & 79.0 & \\
\hline \multicolumn{7}{|c|}{ Healthcare decision-making / Autonomy } \\
\hline Yes & 39.8 & 60.2 & \multirow[t]{2}{*}{$<0.01$} & 32.0 & 68.0 & \multirow[t]{2}{*}{$<0.01$} \\
\hline No & 29.8 & 70.2 & & 26.0 & 74.0 & \\
\hline
\end{tabular}




\begin{tabular}{|c|c|c|c|c|c|c|}
\hline \multirow[t]{2}{*}{ Characteristics } & \multicolumn{3}{|c|}{$\begin{array}{l}\text { Knowledge about hepatitis B } \\
\text { or C }\end{array}$} & \multicolumn{3}{|c|}{$\begin{array}{l}\text { Health behavior about hepatitis } \\
\text { B or C }\end{array}$} \\
\hline & Yes & No & p-value* & Yes & No & p-value* \\
\hline Big problem & 29.1 & 70.9 & \multirow[t]{2}{*}{$<0.01$} & 23.8 & 76.2 & \multirow[t]{2}{*}{$<0.01$} \\
\hline Not a big problem & 38.8 & 61.2 & & 32.8 & 67.2 & \\
\hline \multicolumn{7}{|c|}{ Health insurance coverage } \\
\hline Yes & 41.3 & 58.7 & \multirow[t]{2}{*}{0.07} & 42.4 & 57.6 & \multirow[t]{2}{*}{$<0.01$} \\
\hline No & 34.7 & 65.3 & & 28.9 & 71.1 & \\
\hline
\end{tabular}

With reference to women's health behaviour towards HBV and HCV, some of the respondents (25.7\%) had been tested for HBV and HCV at some point (Table 2).

\section{Association of women's overall knowledge and health behaviour regarding HBV and HCV with respondents' characteristics}

Respondents' overall knowledge about HBV and HCV was found to be high among respondents from Islamabad Capital Territory (56.1\%) and Sindh (41.0\%), those living in urban areas (46.0\%), in the age group of 35 years and above (38.4\%), with more than 10 years of schooling (62.5\%), working in professional, clerical, sales \& services jobs (52.2\%), and belonging to the richest wealth quintile (55.3\%). Respondents having mass media exposure (39.9\%) and autonomy to make decisions about healthcare (39.8\%) indicated better knowledge about HBV and HCV than their counterparts. Furthermore, the overall knowledge about HBV and HCV was found to be high amongst those respondents who had access to distant healthcare facilities for medical services (38.8\%) and health insurance coverage (41.3\%). Similar trends were also found in the results for respondents' health behaviour relating to HBV and HCV.

A statistically significant association $(p<0.05)$ of both variables, i.e. overall knowledge and health behaviour relating to HBV and HCV, was observed with respondents' age, the educational status of respondents and their husbands, the occupation of respondents and their husbands, wealth quintile, exposure to mass media, autonomy to make decisions about healthcare, accessibility of distant healthcare facilities and health insurance coverage.

\section{Bivariate and multivariable logistic regression}

The results of the bivariate and multivariable logistic regression analysis of women's overall knowledge (Table 4) and health behaviour (Table 5) regarding HBV and HCV related to sociodemographic characteristics and covariates are presented below. 
Table 4

Bivariate and multivariable logistic regression models of women's knowledge on hepatitis B or C $(n=$ 12,364)

\begin{tabular}{|c|c|c|c|c|}
\hline \multirow[t]{3}{*}{ Characteristics } & \multicolumn{4}{|c|}{ Knowledge about hepatitis B or C } \\
\hline & Bivariate & & \multicolumn{2}{|c|}{ Multivariable } \\
\hline & $95 \% \mathrm{Cl}$ & $\begin{array}{l}\mathrm{p}- \\
\text { value }\end{array}$ & AOR & $95 \% \mathrm{Cl}$ \\
\hline
\end{tabular}

\section{Regions/provinces}

FATA

Punjab

Sindh

Baluchistan

Khyber Pakhtunkhwa

Islamabad Capital Territory

Geographical classification

Rural

Urban

\section{Respondent's Age}

$15-24$ years

$25-34$ years

35 years and above

\section{Respondent's educational status}

No formal schooling

Up to 5 years of schooling

6-10 years of schooling
1

2.41

$1.70-3.42$

$<0.01$

1.04

$0.71-$

1.51

$3.23 \quad 2.26-4.60<0.01$

1.82

$1.25-$

2.67

1.82

$1.22-2.73$

$<0.01$

1.28

$0.84-$

1.97

$2.08 \quad 1.45-2.98<0.01 \quad 1.29$

$0.88-$

1.88

$\begin{array}{ll}5.92 \quad 3.49- \\ & 10.03\end{array}$

$<0.01 \quad 1.66$

$0.94-$

2.94
0.85

$<0.01$

0.24

0.18

0.08
11

$2.19 \quad 2.02-2.37 \quad<0.01 \quad 1.03$

0.92-

1.14
1

1.65

$1.47-1.86$

$<0.01$

1.49

$1.31-$

1.69

$1.84 \quad 1.64-2.07<0.01$

1.83

$1.61-$

2.09
$<0.01$

$<0.01$

1.60

$1.42-1.79<0.01$

1.39

$1.22-$

1.59

$<0.01$

2.21

$1.99-2.45<0.01$

1.52

$1.33-$

1.74

$<0.01$ 


\begin{tabular}{|c|c|c|c|c|c|c|}
\hline \multirow[t]{3}{*}{ Characteristics } & \multicolumn{6}{|c|}{ Knowledge about hepatitis B or C } \\
\hline & \multicolumn{3}{|c|}{ Bivariate } & \multicolumn{3}{|c|}{ Multivariable } \\
\hline & OR & $95 \% \mathrm{Cl}$ & $\begin{array}{l}\mathrm{p}- \\
\text { value }\end{array}$ & AOR & $95 \% \mathrm{Cl}$ & $\begin{array}{l}\mathrm{p}- \\
\text { value }\end{array}$ \\
\hline More than 10 years of schooling & 5.32 & $4.72-6.01$ & $<0.01$ & 2.66 & $\begin{array}{l}2.25- \\
3.14\end{array}$ & $<0.01$ \\
\hline \multicolumn{7}{|l|}{ Husband's educational status } \\
\hline No formal schooling & 1 & & & 1 & & \\
\hline Up to 5 years of schooling & 1.39 & $1.21-1.59$ & $<0.01$ & 1.09 & $\begin{array}{l}0.94- \\
1.27\end{array}$ & 0.21 \\
\hline $6-10$ years of schooling & 1.75 & $1.57-1.96$ & $<0.01$ & 1.08 & $\begin{array}{l}0.95- \\
1.22\end{array}$ & 0.24 \\
\hline More than 10 years of schooling & 3.62 & $3.21-4.08$ & $<0.01$ & 1.36 & $\begin{array}{l}1.16- \\
1.58\end{array}$ & $<0.01$ \\
\hline \multicolumn{7}{|l|}{ Respondent's occupation } \\
\hline Not working / unemployed & 1 & & & 1 & & \\
\hline $\begin{array}{l}\text { Professional, clerical, sales \& } \\
\text { services }\end{array}$ & 2.02 & $1.71-2.38$ & $<0.01$ & 1.48 & $\begin{array}{l}1.23- \\
1.79\end{array}$ & $<0.01$ \\
\hline Agriculture & 0.37 & $0.31-0.46$ & $<0.01$ & 0.72 & $\begin{array}{l}0.57- \\
0.91\end{array}$ & $<0.01$ \\
\hline Manual or household worker & 0.94 & $0.81-1.08$ & 0.37 & 1.17 & $\begin{array}{l}0.99- \\
1.37\end{array}$ & 0.05 \\
\hline \multicolumn{7}{|l|}{ Husband's occupation } \\
\hline Not working / unemployed & 1 & & & 1 & & \\
\hline $\begin{array}{l}\text { Professional, clerical, sales \& } \\
\text { services }\end{array}$ & 1.89 & $1.52-2.36$ & $<0.01$ & 1.15 & $\begin{array}{l}0.91- \\
1.45\end{array}$ & 0.25 \\
\hline Agriculture & 0.76 & $0.60-0.96$ & 0.02 & 0.91 & $\begin{array}{l}0.71- \\
1.18\end{array}$ & 0.49 \\
\hline Manual or household worker & 1.13 & $0.91-1.40$ & 0.26 & 1.11 & $\begin{array}{l}0.88- \\
1.39\end{array}$ & 0.39 \\
\hline \multicolumn{7}{|l|}{ Wealth quintile } \\
\hline Poorest & 1 & & & 1 & & \\
\hline Poorer & 1.20 & $1.03-1.40$ & 0.02 & 1.18 & $\begin{array}{l}0.99- \\
1.39\end{array}$ & 0.06 \\
\hline
\end{tabular}




\begin{tabular}{|c|c|c|c|c|c|c|}
\hline \multirow[t]{3}{*}{ Characteristics } & \multicolumn{6}{|c|}{ Knowledge about hepatitis B or C } \\
\hline & \multicolumn{3}{|c|}{ Bivariate } & \multicolumn{3}{|c|}{ Multivariable } \\
\hline & OR & $95 \% \mathrm{Cl}$ & $\begin{array}{l}\text { p- } \\
\text { value }\end{array}$ & AOR & $95 \% \mathrm{Cl}$ & $\begin{array}{l}\mathrm{p}- \\
\text { value }\end{array}$ \\
\hline Middle & 1.91 & $1.65-2.21$ & $<0.01$ & 1.61 & $\begin{array}{l}1.34- \\
1.92\end{array}$ & $<0.01$ \\
\hline Richer & 2.47 & $2.15-2.85$ & $<0.01$ & 1.62 & $\begin{array}{l}1.33- \\
1.96\end{array}$ & $<0.01$ \\
\hline Richest & 4.96 & $4.31-5.70$ & $<0.01$ & 2.45 & $\begin{array}{l}1.97- \\
3.04\end{array}$ & $<0.01$ \\
\hline \multicolumn{7}{|c|}{ Exposure to mass media / Access to information } \\
\hline No & 1 & & & 1 & & \\
\hline Yes & 2.09 & $1.91-2.29$ & $<0.01$ & 1.08 & $\begin{array}{l}0.96- \\
1.21\end{array}$ & 0.18 \\
\hline \multicolumn{7}{|c|}{ Healthcare decision-making / Autonomy } \\
\hline No & 1 & & & 1 & & \\
\hline Yes & 1.55 & $1.43-1.68$ & $<0.01$ & 1.22 & $\begin{array}{l}1.11- \\
1.34\end{array}$ & $<0.01$ \\
\hline \multicolumn{7}{|c|}{ Accessibility to distant health facility for seeking medical services } \\
\hline Big problem & 1 & & & 1 & & \\
\hline Not a big problem & 1.54 & $1.42-1.67$ & $<0.01$ & 0.85 & $\begin{array}{l}0.77- \\
0.94\end{array}$ & 0.01 \\
\hline \multicolumn{7}{|c|}{ Health insurance coverage } \\
\hline No & 1 & & & 1 & & \\
\hline Yes & 1.32 & $0.97-1.80$ & 0.07 & 1.16 & $\begin{array}{l}0.82- \\
1.64\end{array}$ & 0.39 \\
\hline
\end{tabular}


Table 5

Bivariate and multivariable logistic regression models of women's health behavior on hepatitis $\mathrm{B}$ or $\mathrm{C}(\mathrm{n}=$ 12,364)

\section{Characteristics}

\section{Health behavior about hepatitis $\mathrm{B}$ or $\mathrm{C}$}

Bivariate

Multivariate

OR $\quad 95 \% \mathrm{Cl} \quad$ p-value AOR $95 \% \mathrm{Cl}$

\section{p-value}

\section{Regions/provinces}

FATA

Punjab

Sindh

Baluchistan

Khyber Pakhtunkhwa

Islamabad Capital Territory

Geographical classification

Rural

Urban

1

2.01

$1.84-$

2.18 $\begin{array}{ll}1.78 \quad 1.27- \\ & 2.50\end{array}$

$2.24 \quad 1.59-$

3.17

$0.94 \quad 0.62-$

1.42

1.18

$0.83-$ 1.68

$\begin{array}{cl}2.64 \quad 1.56- \\ & 4.48\end{array}$

1

$\begin{array}{llll}<.01 & 0.96 & 0.67-1.38 & 0.84\end{array}$

$<0.01$

$1.37 \quad 0.95-1.99$

0.09

0.77

$0.68 \quad 0.44-1.06$

0.08

0.36

0.79

$0.55-1.16$

0.23

0.99

$0.57-1.74$

0.98

\section{Respondent's age}

$15-24$ years

1

1

$25-34$ years

2.08

$1.83-$

2.36

$<0.01$

1.92

$1.68-2.19<0.01$

35 years and above

$\begin{array}{ll}2.07 \quad 1.82 \\ & 2.36\end{array}$

$<0.01$

2.01

$1.74-2.31<0.01$

Respondent's educational status

No formal schooling

Up to 5 years of schooling

6-10 years of schooling

More than 10 years of schooling
1

1.26

$1.11-$

1.42

1.75 1.57-

1.95

$3.37 \quad 2.99-$
1

$<0.01$

1.02

$0.88-1.17$

0.78

$<0.01$

1.20

$1.04-1.38$

0.01

$<0.01$

1.70

$1.43-2.02<0.01$ 


\begin{tabular}{|c|c|c|c|c|c|c|}
\hline \multirow[t]{3}{*}{ Characteristics } & \multicolumn{6}{|c|}{ Health behavior about hepatitis B or C } \\
\hline & \multicolumn{3}{|c|}{ Bivariate } & \multicolumn{3}{|c|}{ Multivariate } \\
\hline & OR & $95 \% \mathrm{Cl}$ & p-value & AOR & $95 \% \mathrm{Cl}$ & p-value \\
\hline \multicolumn{7}{|l|}{ Husband's educational status } \\
\hline No formal schooling & 1 & & & 1 & & \\
\hline Up to 5 years of schooling & 1.53 & $\begin{array}{l}1.33- \\
1.77\end{array}$ & $<0.01$ & 1.28 & $1.10-1.48$ & $<0.01$ \\
\hline $6-10$ years of schooling & 1.66 & $\begin{array}{l}1.48- \\
1.86\end{array}$ & $<0.01$ & 1.14 & $0.99-1.30$ & 0.05 \\
\hline More than 10 years of schooling & 2.93 & $\begin{array}{l}2.58- \\
3.32\end{array}$ & $<0.01$ & 1.34 & $1.14-1.57$ & $<0.01$ \\
\hline \multicolumn{7}{|l|}{ Respondent's occupation } \\
\hline Not working / unemployed & 1 & & & 1 & & \\
\hline $\begin{array}{l}\text { Professional, clerical, sales \& } \\
\text { services }\end{array}$ & 1.75 & $\begin{array}{l}1.48- \\
2.06\end{array}$ & $<0.01$ & 1.29 & $1.07-1.56$ & $<0.01$ \\
\hline Agriculture & 0.52 & $\begin{array}{l}0.43- \\
0.64\end{array}$ & $<0.01$ & 0.88 & $0.70-1.11$ & 0.29 \\
\hline Manual or household worker & 0.98 & $\begin{array}{l}0.84- \\
1.14\end{array}$ & 0.81 & 1.12 & $0.94-1.32$ & 0.19 \\
\hline \multicolumn{7}{|l|}{ Husband's occupation } \\
\hline Not working / unemployed & 1 & & & 1 & & \\
\hline $\begin{array}{l}\text { Professional, clerical, sales \& } \\
\text { services }\end{array}$ & 1.90 & $\begin{array}{l}1.51- \\
2.39\end{array}$ & $<0.01$ & 1.25 & $0.98-1.60$ & 0.07 \\
\hline Agriculture & 0.95 & $\begin{array}{l}0.74- \\
1.22\end{array}$ & 0.71 & 1.04 & $0.80-1.35$ & 0.76 \\
\hline Manual or household worker & 1.09 & $\begin{array}{l}0.87- \\
1.38\end{array}$ & 0.43 & 1.03 & $0.81-1.31$ & 0.81 \\
\hline \multicolumn{7}{|l|}{ Wealth quintile } \\
\hline Poorest & 1 & & & 1 & & \\
\hline Poorer & 1.02 & $\begin{array}{l}0.87- \\
1.19\end{array}$ & 0.81 & 1.06 & $0.89-1.27$ & 0.52 \\
\hline Middle & 1.54 & $\begin{array}{l}1.33- \\
1.80\end{array}$ & $<0.01$ & 1.43 & $1.19-1.72$ & $<0.01$ \\
\hline Richer & 2.21 & $\begin{array}{l}1.91- \\
2.55\end{array}$ & $<0.01$ & 1.62 & $1.33-1.98$ & $<0.01$ \\
\hline
\end{tabular}




\begin{tabular}{|c|c|c|c|c|c|c|}
\hline \multirow[t]{3}{*}{ Characteristics } & \multicolumn{6}{|c|}{ Health behavior about hepatitis B or C } \\
\hline & \multicolumn{3}{|c|}{ Bivariate } & \multicolumn{3}{|c|}{ Multivariate } \\
\hline & $\mathrm{OR}$ & $95 \% \mathrm{Cl}$ & p-value & AOR & $95 \% \mathrm{Cl}$ & p-value \\
\hline Richest & 3.42 & $\begin{array}{l}2.96- \\
3.94\end{array}$ & $<0.01$ & 1.95 & $1.55-2.43$ & $<0.01$ \\
\hline \multicolumn{7}{|c|}{ Exposure to mass media / Access to information } \\
\hline No & 1 & & & 1 & & \\
\hline Yes & 1.84 & $\begin{array}{l}1.67- \\
2.02\end{array}$ & $<0.01$ & 1.01 & $0.89-1.13$ & 0.92 \\
\hline \multicolumn{7}{|c|}{ Healthcare decision-making / Autonomy } \\
\hline No & 1 & & & 1 & & \\
\hline Yes & 1.34 & $\begin{array}{l}1.23- \\
1.46\end{array}$ & $<0.01$ & 0.99 & $0.90-1.09$ & 0.91 \\
\hline \multicolumn{7}{|c|}{ Accessibility to distant health facility for seeking medical services } \\
\hline Big problem & 1 & & & 1 & & \\
\hline Not a big problem & 1.57 & $\begin{array}{l}1.44- \\
1.71\end{array}$ & $<0.01$ & 0.99 & $0.90-1.10$ & 0.98 \\
\hline \multicolumn{7}{|c|}{ Health insurance coverage } \\
\hline No & 1 & & & 1 & & \\
\hline Yes & 1.82 & $\begin{array}{l}1.34- \\
2.47\end{array}$ & $<0.01$ & 1.58 & $1.12-2.21$ & 0.01 \\
\hline
\end{tabular}

Table 4 shows that the geographical classification, husband's occupation, and the regions/provinces (except for Sindh) lose their significance in moving from bivariate to multivariable logistic regression models for overall knowledge about HBV and HCV. The findings indicate that, the older the women are, the better their overall knowledge is. This also applies to respondents' education, with a positive trend of knowledge associated with higher levels of education. However, this trend is not very obvious for husband's educational status, with up to 5 years of schooling and 6-10 years of schooling showing no significant differences compared to people with no formal schooling. Moreover, respondents belonging to the category of professional, clerical, sales \& services (AOR $=1.48,95 \% \mathrm{Cl}: 1.23-1.79$ ) showed greater overall knowledge than those who were not working. Conversely, working in agriculture was negatively associated with knowledge ( $\mathrm{AOR}=0.72,95 \% \mathrm{Cl}$ : 0.57-0.91). The results showed that the richest respondents were more likely to have high overall knowledge about HBV and HCV (AOR $=2.45,95 \% \mathrm{Cl}$ : 1.97-3.04) than the poorest quintile. Moreover, the results indicate that respondents having healthcare autonomy ( $\mathrm{AOR}=1.22,95 \% \mathrm{Cl}: 1.11-1.34)$ were more likely to have comprehensive knowledge about HBV and HCV. 
The results presented in Table 5, related to factors associated with health behaviour, are generally comparable to the results for overall knowledge, showing that older respondents had better health behaviour than women aged 15-24 years. This refers particularly to the relevance of greater age, a higher level of education (this time also for husband's education), and wealth quintile. However, respondents' occupation only showed a significant difference for professions, clerical, sales \& services $(A O R=1.29$, $95 \% \mathrm{Cl}: 1.07-1.56)$ compared to the unemployed. Moreover, the results indicate that respondents with health insurance coverage $(A O R=1.58,95 \% \mathrm{Cl}$ : $1.12-2.21)$ are more likely to display positive health behaviour related to HBV and HCV.

\section{Discussion}

This study set out to explore the relationship of sociodemographic characteristics and other variables with women's overall knowledge and health behaviour related to HBV and HCV. The findings highlight that the majority of women (88.3\%) had heard about HBV and HCV. Nonetheless, only $34.8 \%$ had comprehensive knowledge about how to avoid HBV and HCV. With regard to women's awareness of various ways to avoid HBV and HCV, our study revealed that the majority of women knew that contaminated water and food, as well as use of infected syringes, are risk factors for HBV and HCV. Furthermore, some of the women were also aware of safe sex practices, safe blood transfusions and avoiding contact with infected persons to avoid HBV and HCV. Since the reported percentage of women's awareness is low, these findings highlight the importance of increasing awareness campaigns among the general public and to encourage people to go for testing in order to implement Pakistan's Government Plan to eliminate HBV and HCV by 2030 [2]. These findings are in line with previous research conducted in Ethiopia, which reported that the majority of women had never been screened for HBV [29]. The findings from a study in Ghana revealed that slightly fewer than half of the women surveyed reported knowledge about HBV [30]. Another small-scale study conducted in Pakistan reported that the majority of both men and women $(75.4 \%)$ have poor knowledge regarding HBV and have never gone through HBV testing (96.9\%) [6].

With reference to the association between women's overall knowledge and health behaviour regarding HBV and HCV and sociodemographic characteristics, the present study indicated that women living in urban areas, with higher age, better education, higher income and employed in the group of professional, clerical, sales \& services reported better overall knowledge and health behaviour regarding HBV and HCV. These results are comparable with other studies carried out in Pakistan [11, 31], India [32], Uganda [33] and Poland [34]. Furthermore, these findings are also in line with the framework of social determinants of health, which emphasizes that an individual's socioeconomic position is (positively or negatively) associated with their knowledge and health behaviour [22].

In addition to sociodemographic factors, the findings of this study revealed that exposure to mass media and autonomy to make decisions about personal healthcare were positively associated with good health behaviour related to HBV and HCV. These findings are consistent with the results of previous studies conducted in Poland [34] and Pakistan [35], which highlighted women's autonomy and the positive effect 
of the media on knowledge and awareness about disease prevention. Here, it is also important to note that the media has two important responsibilities in order to play its part in disease prevention. Firstly, it is responsible for creating awareness regarding the causes of disease, available treatments and measures to prevent the disease. Secondly, it is responsible for countering infodemics by avoiding misinformation regarding any health-related issue [36].

Summing up, the findings highlight the importance of obtaining accurate and comprehensive knowledge regarding the prevention and treatment of HBV and HCV. Regarding knowledge and awareness, it is important that reliable and valid knowledge is imparted to married women to bring about change in their attitudes and behaviour in relation to disease [37, 38]. It is evident from various studies that accurate and reliable knowledge helps to reduce myths and doubts associated with HBV and HCV [39-41]. Similarly, reliable sources may be used to disseminate information and knowledge regarding HBV. This information may help in shaping and moulding the attitudes and behaviour of individuals. Therefore, suitable and effective health education programmes and behaviour-change strategies at a community level should be launched in order to reduce misconceptions and help women to access services.

\section{Limitations}

Despite the strengths of this study, such as the large sample size and nationally representative data, one needs to consider several limitations when interpreting the data. A major limitation is the fact that the analysis is based on secondary data, which does not include all the items relevant to a holistic view on the determinants of knowledge and behaviour related to HBV and HCV. Furthermore, the assessment does not distinguish between HBV and HCV and combines these two diseases. Additionally, it is a crosssectional design which does not allow for the investigation of causal relationships.

\section{Conclusion}

This study provides evidence that women's sociodemographic characteristics are associated with knowledge and behaviours relating to HBV and HCV. There is a need to create awareness about the causes and prevention of HBV and HCV among women residing in rural areas and having less education. In order to achieve the goal to eliminate HBV and HCV in Pakistan by 2030, the government needs to launch comprehensive media campaigns, particularly in rural areas, to provide adequate information about HBV and HCV regarding mode of transmission, symptoms of disease and awareness about available treatments and vaccines in order to inculcate favourable attitudes towards seeking treatment and to reduce the spread of infections.

\section{Abbreviations}

AOR: Adjusted odds ratio

Cl: Confidence interval 
HBV: Hepatitis B virus

HCV: Hepatitis C virus

GHSS: Global Health Sector Strategy

KAP: Knowledge, attitude, practice

OR: Odds ratio

PDHS: Pakistan Demographic and Health Survey

SPSS: Statistical Package for the Social Sciences

VIF: Variance inflation factor

WHO: World Health Organization

\section{Declarations}

\section{Ethical Approval and Consent to Participate}

The study is based on a secondary data analysis based on data from PDHS 2017-18, a publicly available dataset. Therefore, an ethical approval is not required. Respondents were informed about the ethics and right of voluntary participation. Written informed consent was obtained.

\section{Consent for Publication}

Not applicable.

\section{Availability of Data and Materials}

Data is publicly available from https://dhsprogram.com/.

\section{Competing Interest}

The authors declare that no competing interest exist. FF serves on the Editorial Board of BMC Public Health as Associate Editor.

\section{Funding}


This research received no supporting funds from any funding agency in the public, commercial, or not-forprofit sector.

\section{Authors Contributions}

SM and RZ conceptualized the study. SM and SI analysed the data. RZ, MZZ, and FF supervised the work and supported in data analysis. SM drafted the draft, SI, RZ, MZZ, and FF revised it critically for important intellectual content. All auhtors approved the final manuscript.

\section{Acknowledgements}

We acknowledge support from the German Research Foundation (DFG) and the Open Access Publication Fund of Charité - Universitätsmedizin Berlin.

\section{References}

1. Shawa IT. Hepatitis B and C viruses. IntechOpen. 2019; doi: 10.5772/intechopen.82772.

2. WHO Regional Office for the Eastern Mediterranean. Pakistan - Prevention and control of hepatitis. 2020. http://www.emro.who.int/pak/programmes/prevention-a-control-of-hepatitis.html. Accessed 17 September 2020.

3. Waheed Y, Shafi T, Safi, SZ, Qadri I. Hepatitis C virus in Pakistan: a systematic review of prevalence, genotypes and risk factors. World J Gastroenterol. 2009;15(45):5647-53.

4. Munaf A, Memon MS, Kumar P, Ahmed S, Kumar MB. Comparison of viral hepatitis-associated hepatocellular carcinoma due to HBV and HCV - cohort from liver clinics in Pakistan. Asian Pac J Cancer Prev. 2014;15(18):7563-7.

5. Lim AG, Qureshi H, Mahmood H, Hamid S, Davies CF, Trickey A, et al. Curbing the hepatitis C virus epidemic in Pakistan: the impact of scaling up treatment and prevention for achieving elimination. Int J Epidemiol. 2018;47(2):550-60.

6. ul Haq N, Hassali MA, Shafie AA, Saleem F, Farooqui M, Haseeb A, Aljadhey H. A cross-sectional assessment of knowledge, attitude and practice among Hepatitis-B patients in Quetta, Pakistan. BMC Public Health. 2013;13:448.

7. Leung N. Chronic hepatitis B in Asian women of childbearing age. Hepatol Int. 2009;3(Suppl. 1):2431.

8. Noreen N, Kumar R, Shaikh BT. Knowledge about hepatitis B vaccination among women of childbearing age: a cross-sectional study from a rural district of Punjab, Pakistan. East Mediterr Health J. 2015;21(2):129-33.

9. Nankya-Mutyoba J, Aizire J, Makumbi F, Ocama P, Kirk GD. Hepatitis B virus perceptions and health seeking behaviors among pregnant women in Uganda: implications for prevention and policy. BMC 
Health Serv Res. 2019;19:760.

10. Yelemkoure ET, Yonli AT, Montesano C, Ouattara AK, Diarra B, Zohoncon TM. Prevention of mother-tochild transmission of hepatitis B virus in Burkina Faso: screening, vaccination and evaluation of postvaccination antibodies against hepatitis B surface antigen in newborns. J Public Health Afr. 2018;9(3):816.

11. Talpur AA, Memon NA, Solangi RA, Ghumro AA. Knowledge and attitude of patients towards hepatitis B and C. Pak J Surg. 2007;23(3):162-5.

12. Khan N, Ahmed SM, Khalid MM, Siddiqui SH, Merchant AA. Effect of gender and age on the knowledge, attitude and practice regarding hepatitis $B$ and $C$ and vaccination status of hepatitis $B$ among medical students of Karachi. Pakistan. J Pak Med Assoc. 2010;60(6):450-5.

13. Alam M, Tariq WUZ. Knowledge attitudes and practices about Hepatitis $B$ and $C$ among young healthy males. Pakistan Journal of Pathology. 2006;17(4):147-50.

14. Ross J, Smith DP. Korea-trends in 4 National KAP Surveys, 1964-67. Stud Fam Plan. 1969;43:6-11.

15. A conceptual framework for action on the social determinants of health. Geneva: World Health Organization; 2010.

16. Ezeruigbo Chinwe R, UdeNebonta Abigail R. Impact of health education on knowledge, attitude and practice of cervical cancer screening among secondary school teachers in Enugu State. Journal of Women's Health Care. 2015;4:1-7.

17. Nganwai P, Truadpon P, Inpa C, Sangpetngam B, Mekjarasnapa M, Apirakarn M, Chumworathayi B. Knowledge, attitude and practice vis-à-vis cervical cancer among registered nurses at the faculty of medicine Khon Kaen University, Thailand. Asian Pac J Cancer Prev. 2008;9(1):15-18.

18. Gimenez-Sanchez F, Butler JC, Jernigan DB, Strausbaugh LJ, Slemp CC, Perilla MJ, Dowell SF. Treating cardiovascular disease with antimicrobial agents: a survey of knowledge, attitudes, and practices among physicians in the United States. Clin Infect Dis. 2001;33(2):171-6.

19. Bansal AB, Pakhare AP, Kapoor N, Mehrotra R, Kokane AM. Knowledge, attitude, and practices related to cervical cancer among adult women: a hospital-based cross-sectional study. J Nat Sci Biol Med. 2015;6(2):324-8.

20. Khalil A, Abdalrahim M. Knowledge, attitudes, and practices towards prevention and early detection of chronic kidney disease. Int Nurs Rev. 2014;61(2):237-45.

21. Rana M, Sayem A, Karim R, Islam N, Islam R, Zaman TK, Hossain G. Assessment of knowledge regarding TB among non-medical university students in Bangladesh: a cross-sectional study. BMC Public Health. 2015;15:716.

22. Blas E, Sommerfeld J, Sivasankara Kurup A. Social determinants approaches to public health: from concept to practice. Geneva: World Health Organization; 2011.

23. Arrivillaga $M$, Ross $M$, Useche $B$, Alzate ML, Correa $D$. Social position, gender role, and treatment adherence among Colombian women living with HIV/AIDS: social determinants of health approach. Rev Panam Salud Publica. 2009;26(6):502-10. 
24. Poundstone KE, Strathdee SA, Celentano DD. The social epidemiology of human immunodeficiency virus/acquired immunodeficiency syndrome. Epidemiol Rev. 2004;26:22-35.

25. Roberts KJ, Mann T. Barriers to antiretroviral medication adherence in HIV-infected women. AIDS Care. 2000;12(4):377-86.

26. Ickovics JR, Meade CS. Adherence to HAART among patients with HIV: breakthroughs and barriers. AIDS Care. 2002;14(3):309-18.

27. Ahmad A, Sann LM, Rahman HA. Factors associated with knowledge, attitude and practice related to hepatitis B and C among international students of University Putra Malaysia. BMC Public Health. $2016 ; 16,611$.

28. Shaghaghian S, Pardis S, Mansoori Z. Knowledge, attitude and practice of dentists towards prophylaxis after exposure to blood and body fluids. Int J Occup Environ Med. 2014;5(3):146-54.

29. Gebrecherkos T, Girmay G, Lemma M, Negash M. Knowledge, attitude, and practice towards Hepatitis $B$ virus among pregnant women attending antenatal care at the University of Gondar comprehensive specialized hospital, Northwest Ethiopia. Int J Hepatol. 2020; doi: 10.1155/2020/5617603.

30. Dun-Dery F, Adokiya MN, Walana W, Yirkyio E, Ziem JB. Assessing the knowledge of expectant mothers on mother-to-child transmission of viral hepatitis B in Upper West region of Ghana. BMC Infect Dis. 2017;17:416.

31. Awan FR, Baig MS. Knowledge Of Hepatitis Prevention Among Reproductive Age Group Women From Pakistan Demographic Health Survey Data 2012-13. Pakistan Journal of Public Health. 2019;9(3):135-8.

32. Mukherjee PS, Dutta E, Das DK, Ghosh S, Neogi S, Sarkar A. Knowledge about hepatitis B and hepatitis $C$ virus infection and consequences: a cross-sectional assessment of baseline knowledge among infected patients in West Bengal, India. Hepatol Med Policy. 2017;2:6.

33. Nankya-Mutyoba J, Aizire J, Makumbi F, Atuyambe L, Ocama P, Kirk GD. Correlates of hepatitis B awareness and disease-specific knowledge among pregnant women in Northern and Central Uganda: a cross-sectional study. Hepatol Med Policy. 2018;3:14.

34. Ganczak M, Dmytrzyk-Daniłów G, Korzeń M, Drozd-Dąbrowska M, Szych Z. Prevalence of HBV infection and knowledge of hepatitis $B$ among patients attending primary care clinics in Poland. $J$ Community Health. 2016;41(3):635-44.

35. Iqbal S, Maqsood S, Zafar A, Zakar R, Zakar MZ, Fischer F. Determinants of overall knowledge of and attitudes towards HIV/AIDS transmission among ever-married women in Pakistan: evidence from the Demographic and Health Survey 2012-13. BMC Public Health. 2019;19:793.

36. Islam MS, Sarkar T, Khan SH, Mostofa Kamal AH, Hasan SMM, Kabir A, et al. COVID-19-Related Infodemic and Its Impact on Public Health: A Global Social Media Analysis. Am J Trop Med Hyg. 2020; doi: 10.4269/ajtmh.20-0812.

37. Bandura A. Health promotion by social cognitive means. Health Educ Behav. 2004;31(2):143-64.

38. Anderson ES, Wagstaff DA, Heckman TG, Winett RA, Roffman RA, Solomon LJ, Cargill V, Kelly JA, Sikkema KJ. Information-motivation behavioral skills (IMB) model: testing direct and mediated 
treatment effects on condom use among women in low-income housing. Ann Behav Med. 2006;31(1):70-9.

39. Rajamoorthy Y, Taib NM, Munusamy S, Anwar S, Wagner AL, Mudatsir M, et al. Knowledge and awareness of hepatitis B among households in Malaysia: a community-based cross-sectional survey. BMC Public Health. 2019;19.47.

40. Ni YH, Huang LM, Chang MH, Yen CJ, Lu CY, You SL, et al. Two decades of universal hepatitis B vaccination in Taiwan: impact and implication for future strategies. Gastroenterology. 2007;132(4):1287-93.

41. Qureshi H, Bile KM, Jooma R, Alam SE, Afridi HUR. Prevalence of hepatitis B and C viral infections in Pakistan: findings of a national survey appealing for effective prevention and control measures. East Mediterr Health J. 2010;16(Suppl.):15-23.

\section{Figures}

$50.0 \%$

$40.0 \%$

$30.0 \%$

$20.0 \%$

$17.9 \%$
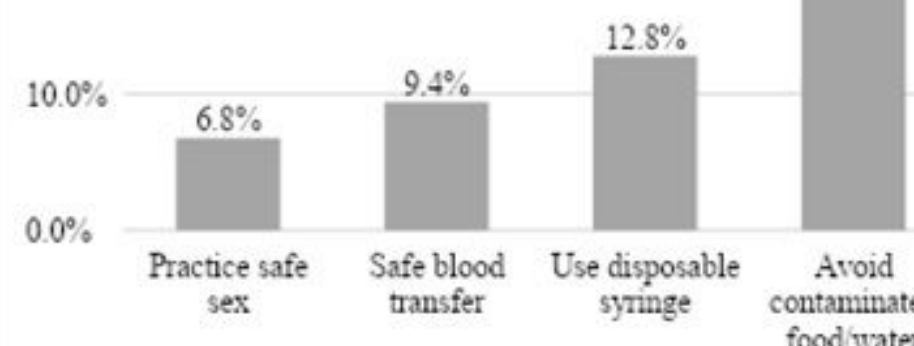

Avoid contaminated food water

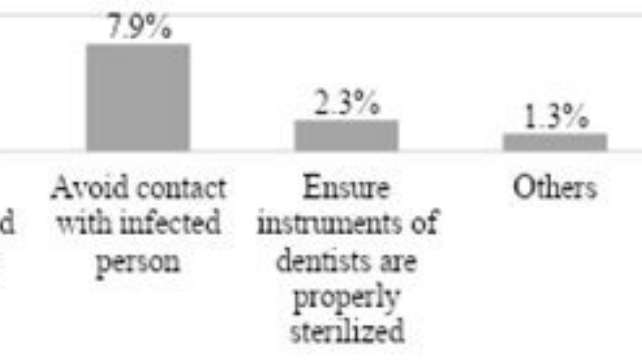

Figure 1

Awareness about various ways to avoid hepatitis B or C 